\title{
La praxis educativa: una aproximación a la realidad en el aula
}

\author{
Castro, Elizabeth * \\ Peley, Rosario ** \\ Morillo, Roselia ***
}

\section{Resumen}

Es inquietante observar en las escuelas, grupos de alumnos apáticos y desanimados en sus estudios, pero lo más alarmante de esta realidad, no sólo es la falta de interés de los alumnos, sino también de los docentes que tienen la responsabilidad y el deber de dirigir y administrar la educación en el aula, al igual que tienen la responsabilidad de desarrollar el proceso motivacional del alumno, y guiarlo de manera pertinente. En este sentido, el presente artículo analiza la praxis educativa en el aula y como se aplican las estrategias instruccionales que el docente utiliza en la actualidad durante el proceso instruccional. La información fue recabada a través de las técnicas de la observación, la entrevista a profundidad y un cuestionario. Los resultados reflejan que un alto porcentaje de docentes utilizan estrategias instruccionales, planifican sus clases y se consideran altamente responsables, mientras que los porcentajes mas bajos los arrojan las categorías orientadas a la participación y retroalimentación en el aula, sin embargo estos resultados permitieron concluir que la práctica pedagógica del docente en la utilización de estrategias instruccionales despiertan muy poco el deseo de aprender por parte de los alumnos, así como el desarrollo de las capacidades innovadoras, igualmente se evidencia que los docentes no promueven durante el desarrollo de sus clases la participación activa y consciente de sus alumnos.

Palabras clave: Praxis educativa, estrategias instruccionales, aprendizaje significativo.

Recibido: 21-02-08 • Aceptado: 22-01-09

* Profesora Titular de la Escuela de Educación, Facultad de Humanidades y Educación de la Universidad del Zulia. Licenciada en Educación. Magister en Gerencia Educativa. Doctora en Ciencias Gerenciales. E-mail: lizcas8@gmail.com

** Profesora Asociada de la Escuela de Educación, Facultad de Humanidades y Educación de la Universidad del Zulia. Magíster en Planificación Educativa. E-mail: rospeley@intercable.net.ve

*** Profesora Titular de la Escuela de Educación, Facultad de Humanidades y Educación de la Universidad del Zulia. Doctora en Ciencias Humanas. E-mail: roseliamorillo@yahoo.com 
La praxis educativa: una aproximación a la realidad en el aula

Castro, Elizabeth; Peley, Rosario y Morillo, Roselia

\title{
Educational Praxis: An Approach to Reality in the Classroom
}

\begin{abstract}
It is disturbing to see groups of students in schools who are apathetic and discouraged in their studies, but the most alarming thing about this reality is not the students' lack of interest but that of the teachers who have the responsibility and duty to direct and administer education in the classroom, as well as having the responsibility for developing the student's motivation and guiding him/her in an appropriate manner. This article analyzes the practice of education in the classroom and how instructional strategies that teachers currently use during the instructional process are applied. The information was collected through the techniques of observation, in-depth interviews and a questionnaire. Data collection instruments utilized were screen play observation, teacher questionnaires and interview scripts. Findings reflect that a high percentage of teachers use instructional strategies, plan their classes and consider themselves to be highly responsible, while the lowest percentages are found in categories oriented toward participation and feedback in the classroom. These results permit concluding that the teacher's pedagogical practice in utilizing instructional strategies awakens very little desire for learning on the part of the students, or for the development of innovative capacities; it is also shown that the teachers do not promote active and conscious participation by their students during classes.
\end{abstract}

Key words: Educational praxis, instructional strategies, meaningful learning.

\section{Introducción}

Vivimos un período caracterizado por una constante renovación de los conocimientos. Algunos autores han llegado a afirmar que cada doce años se está duplicando el conocimiento humano. En estas condiciones, es absurdo pretender que la escuela siga concentrada en los aprendizajes memorísticos. Más que el conocimiento, se torna como prioritaria la capacidad para comprenderlo, para interpretarlo y procesarlo. Frente a una escuela concentrada en el aprendizaje de informaciones particulares, el mundo contemporáneo exige la formación de individuos con mayor capacidad de observación, análisis y síntesis.

En tal sentido, para conseguir ese individuo crítico, creativo, innovador y creador de soluciones a los problemas de su entorno, se requiere la utilización de un factor determinante como lo son las estrategias instruccionales empleadas por el docente, que le permitan adaptar su quehacer docente a los avances del conocimiento científico, técnico y pedagógico que garanticen una actuación rigurosa, sistemática, reflexiva y coherente tanto en el centro educativo como en la propia aula.

En este sentido, el presente artículo analiza la praxis educativa en el aula y como se aplican las estrategias instruccionales que el docente utiliza en la actualidad durante el proceso instruccional. La información fue recabada a través de las técnicas de la observación, la entrevista a profundidad y un cuestionario. Los instrumentos para la recolección de datos que 
se utilizaron fueron el Guión de observación, los Cuestionarios a docentes y el Guión de entrevista. Para el análisis de los datos se utilizó la técnica de la triangulación para comparar la diversidad de resultados obtenidos a través de las diferentes técnicas utilizadas. Esta técnica se empleó con el propósito de poder contrastar las opiniones y respuestas obtenidas de la muestra, a través de los cuestionarios, las observaciones y entrevistas realizadas, con el fin de obtener un mayor porcentaje de validez en los resultados de la investigación. La población estuvo constituida por los docentes que administran las diferentes áreas académicas en la primera y segunda etapa del Nivel de Educación Básica pública.

\section{Algunas concepciones sobre la educación}

El estudio de los fenómenos educativos y el ejercicio de la docencia, puede plasmarse desde múltiples aproximaciones disciplinarias, dada la complejidad que presentan no solo la explicación de los procesos de aprendizaje y desarrollo personal involucrado, sino la necesidad de disponer tanto de un marco de referencia interpretativo como estrategia de intervención específica que le permita orientar la reflexión y la práctica. Castillo (1996) expone que, el desarrollo de la educación debe partir de un enfoque dirigido al docente, con el fin de aprovechar al máximo su motivación, experiencia, habilidad en el tratamiento de las situaciones educativas y la voluntad de continuar en un proceso de autorrealización y mejoramiento permanente.
A este respecto, Ausubel (1983), señala que la educación es un proceso mediante el cual el individuo desarrolla sus habilidades físicas, intelectuales y morales bajo los lineamientos sociopolíticos de cada país, para mantener, en el tiempo y en el espacio, los principios filosóficos de cada sociedad. Bajo este contexto, la Educación Básica tiene como finalidad la formación integral del educando. Por lo tanto, debe atender a todas las áreas de su personalidad: conocimientos, habilidades, destrezas, valores y aptitudes.

La educación contemporánea en contraposición con la tradicional tiene como función principal orientar lo que el hombre manifiesta hacia la plenitud de actualización y expansión, por lo que las estrategias deben permitir a los alumnos actuar conscientemente frente a diversas situaciones de la vida, aprovechando las experiencias, integración de las ideas y progreso social.

Sin embargo, a pesar de algunos intentos por incorporar políticas transformadoras, aún persiste en el nivel de Educación Básica en Venezuela un estado de crisis dado que la educación formal que se imparte muestra poca vinculación con las prioridades y las expectativas que el país ha colocado en este sector, como instrumento de formación y transformación que reclama la sociedad actual, razón por la cual el docente constituye un componente imprescindible para lograr una educación de calidad, por lo tanto, su formación debe estar adaptada a las demandas personales de los alumnos y sociales de la comunidad, a la evolución científico - tecnológica y al ritmo cambian- 
La praxis educativa: una aproximación a la realidad en el aula

Castro, Elizabeth; Peley, Rosario y Morillo, Roselia

te de la cultura, valores y comunicación de la sociedad donde se desenvuelve.

En el nivel de Educación Básica, el aprendizaje de los alumnos depende en gran medida de la actitud del docente, de su preparación académica y de la forma como organice las actividades pedagógicas. Esto es de particular significación en la I y II Etapa, sobre todo en los primeros grados del nivel, donde los índices de repitencia y deserción son más elevados; situación que demanda especial atención (Rivero, 1995).

El modelo educativo actual enfatiza que los estudiantes se involucren activamente, reflexionen sobre sus aprendizajes, hagan inferencias y experimenten conflictos cognitivos, de allí que, es necesario introducir cambios en los procedimientos que se utilizan en las aulas.

Cabe mencionar que el Currículo Básico Nacional (1997), tiene entre sus bases el constructivismo, puesto que éste ha incorporado una serie de estrategias de planificación, dentro de las cuales se encuentran los Proyectos Educativos Integrales Comunitarios (PEIC) y los Proyectos Pedagógicos de Aula (PPA), los cuales apoyan la gestión y autonomía de los planteles y fomentan la integración de los aprendizajes. Esta propuesta de acción tiene como objetivo elevar la calidad de la educación y la transformación de las prácticas pedagógicas hacia el perfeccionamiento de la formación, ética e intelectual de los alumnos, mediante un conjunto de acciones planificadas por los miembros de la comunidad educativa, orientadas a resolver los problemas pedagógicos de la escuela, a través de los proyectos propuestos por el Ministerio de Educación Cultura y Deporte (MECD).

\section{El proceso instruccional en el aula}

En el proceso instruccional juega un papel importante las estrategias instruccionales que el docente aplica en el aula, debido a que, las estrategias instruccionales constituyen el conjunto de orientaciones didácticas que señalan, en forma clara e inequívoca, los métodos, procedimientos, técnicas y recursos que se planifican para el logro de todos y cada uno de los aprendizajes contemplados en los objetivos instruccionales. Díaz y Pacheco (1989).

Específicamente, las estrategias instruccionales indican las actividades, ejercicios, problemas o cualquier tipo de experiencia por parte del docente o el alumno que tornen más efectivo el proceso de enseñanza-aprendizaje y faciliten la consecución de los objetivos. Éstas, deben estar en concordancia con las características, intereses, necesidades, expectativas y motivaciones del alumno, y al propio tiempo, responder a la simplificación del esfuerzo, a la eficacia y a la utilización de criterios de selección fundamentados en la naturaleza de la asignatura y los objetivos establecidos.

Para Díaz y Pacheco (1989), las estrategias instruccionales, atendiendo al uso, pueden clasificarse como siguen:

1. Estrategias Cognoscitivas: las cuales facilitan la construcción, la aplicación y la validación del conocimiento, con variedad de propósitos, tales como la solución de problemas, la exploración de conocimientos, y en general, la realización de cualquier acto mental.

2. Estrategias Metacognoscitivas: las cuales especifican los pasos para su- 
pervisar, evaluar y mejorar cualquier pensamiento o acción que se esté ejecutando.

3. Estrategias Didácticas: éstas especifican las secuencias de actividades a realizar para organizar y conducir el proceso de enseñanza - aprendizaje. Razón por la cual, el docente, al momento de planificar sus actividades debe considerar el tipo de estrategias que mejor se adapte a los objetivos y contenidos a tratar.

El trabajo del alumno plantea tres grupos de clasificación de las estrategias instruccionales: las individuales, cuando al tratar de minimizar las diferencias individuales el trabajo de aula se adecua al alumno; las colectivas, donde todos los alumnos cooperan con el trabajo; y las mixtas, en el desarrollo de las cuales se planifican actividades individuales y socializadas.

Otro aspecto señalado por Nérice (1980), es la aceptación de lo enseñado, ubica en esta categoría, las estrategias dogmáticas, donde la acción del docente se concentra en la transmisión, de manera autoritaria, de contenidos y, las heurísticas, cuando su práctica pedagógica va dirigida a estimular y guiar el aprendizaje.

Por su parte, Flórez (1996), clasifica las estrategias instruccionales considerando el agente, en: magistrales, las cuales requieren de la intervención del docente dirigida al alumno o al grupo; socializadas, referidas a aquellas estrategias instruccionales en las que el docente y los alumnos forman un grupo de aprendizaje, e individualizadas requieren de la actividad personal del alumno de acuerdo con sus intereses y según sus aptitudes, actitudes y ritmo de aprendizaje.
Por otra parte, el proceso instruccional, implica además, considerar la comunicación, los mensajes emitidos por el docente, el clima en el aula, entendido el clima como el ambiente psicológico que afecta al grupo, así como, la participación libre y espontánea de los alumnos, en el cual el papel del maestro en su praxis, está orientado a generar un clima de confianza, de respeto a las ideas y de tolerancia hacia las opiniones de los miembros en el aula.

\section{Categorías de análisis consideradas para el estudio de la praxis educativa}

Una vez procesada la información que se logró obtener por medio de la aplicación de los instrumentos de recolección de datos, se analizaron e interpretaron los resultados por categorías de análisis.

- Categoría: Estrategias instruccionales utilizadas por los docentes.

Un $85.71 \%$ de los docentes utilizan mayormente estrategias instruccionales pasivas. En relación a este aspecto, se puede afirmar que según los resultados no se estimula un aprendizaje permanente creativo que le permita generar acciones que conlleven la transformación de situaciones inmediatas de su entorno.

Con respecto a la misma categoría, el guión de observación reveló que sólo un $35 \%$ de los docentes utiliza estrategias instruccionales activas. Este bajo porcentaje ayuda a entender el porque en el aula se presentan pocas oportunidades de aprendizaje que favorezcan en el alumno vivenciar situaciones de aprendizaje significativo. Olvidándose de afirma- 
La praxis educativa: una aproximación a la realidad en el aula Castro, Elizabeth; Peley, Rosario y Morillo, Roselia

ciones relevantes como las realizadas por Gagné (1987), en las cuales señala, que las variaciones del ritmo, tiempo y metas que los docentes emplean para mantener la organización de la clase, deben ser más de tipo dinámico que mecánico, es decir, que muy poco gana el docente si se dedica al uso de estrategias instruccionales pasivas.

De las informaciones obtenidas se infiere que los docentes emplean estrategias instruccionales pasivas aun y cuando contradictoriamente aseguran que no las utiliza, como se observó en los resultados del cuestionario, un $78.57 \%$ afirman que siempre utilizan actividades innovadoras y el otro $21.42 \%$ restante afirmaron que frecuentemente utilizan una planificación innovadora para el desarrollo de sus clases, es decir, que un $99.99 \%$ de los docentes encuestados expresan utilizar actividades dinámicas e innovadoras en sus planificaciones.

Esto coincide con lo manifestado por los informantes claves durante la entrevista, en las cuales se obtuvieron los siguientes resultados.

"En las estrategias instruccionales activas yo ubico las dinámicas de grupo, también utilizo mucho las de transparencias, repasado y muchas otras que ahorita no recuerdo, pero de verdad que son muchas" (docente1).

"Las estrategias que yo uso dependen de lo que ellos me pidan, hay veces que vienen todos tristones, entonces quieren trabajar, como le explico, más que todo escrito, podemos decir que utilizo a veces estrategias instruccionales pasivas y a veces activas" (docente 3 ).

Como se puede observar, con este tipo de respuestas los docentes contradi- cen su actuación, puesto que lo expresado en el cuestionario no coincide con lo observado y constatado durante la observación de sus clases y en la entrevista a profundidad.

Por otra parte, se observó que un $92.85 \%$ utilizan estrategias instruccionales individuales, un $64.28 \%$ las estrategias instruccionales colectivas y sólo un $50 \%$ las estrategias instruccionales mixtas. Esto se refleja en el resultado arrojado por la investigación, puesto que los docentes en el cuestionario afirmaron en un $71.42 \%$ utilizar siempre estrategias instruccionales actualizadas en la planificación de sus clases, y el $28.57 \%$ restante las utilizan frecuentemente. Lo cual evidencia un menor uso de las estrategias instruccionales mixtas como actualizadas, es decir, que el docente se inclina marcadamente por el uso de las estrategias instruccionales individuales en detrimento de las mixtas, en contraposición a lo que señala Nérice (1980) ya que para propiciar un aprendizaje significativo las actividades de los alumnos deben estar cargadas de esos tres tipos de estrategias, sin establecer discriminaciones entre estos, debido a que al incluir en el proceso enseñanza-aprendizaje las estrategias instruccionales individuales, colectivas y mixtas, se están brindando al alumno mayores oportunidades para desarroIlar habilidades y destrezas cognoscitivas, en este sentido, se estaría mediando el proceso enseñanza-aprendizaje desde diferentes perspectivas, logrando así el docente optimizar el proceso.

El análisis de los resultados obtenidos a través de los docentes entrevistados demuestra que existe un desinterés por el uso de estrategias instruccionales 
mixtas, ya que, los docentes respondieron al respecto lo siguiente.

"Yo utilizo muy poco las estrategias instruccionales mixtas, utilizo más que todo las grupales" (docente 4).

"Las estrategias instruccionales mixtas las utilizo muy poco, porque no me gustan las individuales, me gusta que todos aprendan a la vez" (docente 5).

"Con el uso de estrategias instruccionales mixtas me siento un poco incomoda, siento que los alumnos no aprenden igual que con las individuales, yo utilizo más las individuales, porque yo creo que allí entran las evaluaciones" (docente 6).

El $100 \%$ de los docentes observados hizo uso en algún momento de la clase de estrategias instruccionales dogmáticas, queriendo transmitir los contenidos de manera autoritaria, debido en ocasiones, al mal comportamiento de los alumnos, o por la falta de tiempo de los docentes para el desarrollo de la clase. Al utilizar el docente dichas estrategias se coharta en el alumno la oportunidad de recibir aprendizaje significativo, puesto que la actuación autoritaria por parte del docente anula toda posibilidad de gestar espacios oportunos dentro del aula que estimulen al alumno a desarrollar sus habilidades y destrezas cognoscitivas.

Con respecto al uso de estrategias cognoscitivas, se muestra, que el $14.28 \%$ de los docentes utilizan dichas estrategias; las cuales requieren atención e interés en todas las etapas que se buscan abarcar con este tipo de estrategias, como lo son la construcción del conocimiento, el acompañamiento por parte del docente en la aplicación de este y por último, pero no menos importante, la valida- ción del conocimiento. En lo que respecta a esta última etapa de las estrategias instruccionales cognoscitivas, se observó que el $78.57 \%$ de los docente utilizaron estrategias de apoyo, con la finalidad de que sus alumnos reforzaran los conocimientos e información suministrada (validación del conocimiento), facilitando así el aprendizaje.

En relación al uso de estrategias metacognoscitivas un $14.28 \%$ hace uso de estas, es decir, que del total de los maestros observados sólo dos de ellos se preocuparon en guiar a sus alumnos por los diferentes pasos con que cuentan dichas estrategias para conseguir los resultados deseados, en otras palabras, el docente no supervisa ni evalúa el proceso de enseñanza aprendizaje, obviando la facilitación para mejorar cualquier acción que el alumno esté ejecutando. Estos resultados se ratifican cuando los docentes manifiestan respuestas como las siguientes en la entrevista realizada.

"Esas no sé cuales son, nunca las he oído" (docente 2).

"¿Estrategias metacognoscitivas?.., no, no estoy segura de cuales son esas estrategias, creo que nunca las he utilizado, o tal vez si pero sin darme cuenta, tu sabes que son tantas que uno se confunde, o se olvidan con el tiempo" (docente 6).

"No utilizo ese tipo de estrategias porque no ayudan mucho a los alumnos. Tu sabes, con ellos hay que ser muy concretos" (docente 11).

Evidenciándose en las afirmaciones anteriores un desconocimiento conceptual, restringiendo y dificultando la adecuada utilización de las estrategias metacognoscitivas. 
La praxis educativa: una aproximación a la realidad en el aula Castro, Elizabeth; Peley, Rosario y Morillo, Roselia

En lo que respecta al uso de estrategias didácticas el guión de observación permitió detectar que un $87.71 \%$ hace uso de estas estrategias, puesto que los docentes en su mayoría le indican a sus alumnos la actividad a realizar, aun cuando no especifican la secuencia a seguir durante la ejecución de la misma, es decir, la práctica pedagógica se desarrolla bajo un ambiente poco participativo por parte del alumno, representado por estrategias magistrales debido a los escasos recursos con los que cuenta la institución, y la poca preparación del docente en lo que respecta a las estrategias didácticas que promueven aprendizaje significativo.

Para el uso de estrategias de procedimiento, se observó que el $64.28 \%$ de los docentes hace uso de ellas, permitiendo a los alumnos aprender de sus experiencias, es decir, que este tipo de estrategias conllevan a desarrollar con los alumnos procedimientos y competencias necesarios para impartir los conocimientos, esto es, basar el trabajo en el aprendizaje de procedimientos y actitudes más que en la transmisión de nociones.

La ausencia de estas estrategias contradice lo explicitado en el cuestionario por los docentes, donde un $78.57 \%$ afirmaron manejar la planificación de los PPA, es decir, que a diferencia de lo realmente observado, la mayoría de los docentes encuestados manifiesta tener presente la planificación planteada en los proyectos, que amerita por su naturaleza la consideración de estrategias de personalidad, puesto que en los PPA se busca la participación activa del alumno, y las estrategias de personalidad brindan la oportunidad de producir nuevas ideas, nuevos enfoques, despertando así el interés en los alumnos de participar en la construcción de dichos proyectos.

En relación al uso de estrategias instruccionales durante los diferentes momentos de la clase, los resultados fueron los siguientes: En cuanto al uso de estrategias preinstruccionales, un $35.71 \%$ hizo uso de ellas, evidenciándose así que los docentes no le brindan importancia a este tipo de estrategias instruccionales.

Estos resultados permiten afirmar que los docentes no se preocupan por insertar al alumno en la actividad a iniciar, ni por comunicarles el objetivo, propósito o competencia que se pretende cubrir con el desarrollo de esa actividad, evitando así que la nueva información pueda relacionarse de modo no arbitrario y sustancial con lo que el alumno ya sabe. Cuando se logra una adecuada relación entre lo existente y lo nuevo, el alumno se siente orientado, pudiendo así percibir de mejor manera la intencionalidad del docente durante el desarrollo de la clase a través del nuevo contenido, destacando que cuando el material no se suministra arbitrariamente se logra una mejor comprensión y disposición hacia lo nuevo, puesto que un mismo concepto o proposición puede expresarse de manera sinónima y seguir transmitiendo exactamente el mismo significado.

Tal y como lo señalan Díaz y Hernández (2001) al expresar que ninguna tarea de aprendizaje se realiza en el vacío cognitivo, aun tratándose de aprendizajes repetitivos o memorísticos, este puede relacionarse con la estructura cognitiva aunque sea arbitrariamente y sin adquisición de significado. 
En cuanto al uso de estrategias coinstruccionales, el porcentaje de docentes que las utilizan se ubicó en un $85.71 \%$, puesto que en la mayoría de las observaciones el docente orientó la ejecución de las actividades desarrolladas con los alumnos, logrando así en la práctica estructurar e interrelacionar los contenidos, contribuyendo a mantener la atención durante la presentación de ilustraciones, comparaciones e incluso procesos más complejos como lo constituye el de enseñarles a leer y escribir.

La baja utilización de estrategias preinstruccionales (35.71\%) y la persistencia del uso de las estrategias coinstruccionales (85.71\%), contribuyeron a develar que el $92.81 \%$ de los docentes no hace uso de las estrategias postinstruccionales, las cuales son importantes para solidificar o reafirmar los conocimientos de los alumnos, y poder constatar así el aprendizaje o captación del tema o actividad tratada, sólo un docente utilizó estrategias postinstruccionales.

Es oportuno destacar que en el proceso enseñanza-aprendizaje es fundamental que estos tres tipos de estrategias (preintruccionales, coinstruccionales $y$ postinstruccionales) acompañen el desarrollo de la clase para que se pueda gestar un aprendizaje significativo en el grupo de alumnos, ya que, al utilizar las estrategias preinstruccionales se le brinda al alumno la oportunidad de demostrar sus habilidades y destrezas y poder luego, en el transcurso de la clase, enriquecerlas. Al utilizar los tres tipos de estrategias instruccionales mencionadas, se inserta al alumno en el conocimiento procedimental, al igual que se le ayuda como lo señala Sánchez (1999:9) "[en] su operacionalización y con- creción en el desarrollo de habilidades y destrezas cognoscitivas".

La información suministrada por los docentes es congruente con lo expresado en la entrevista por los informantes claves que dieron respuestas como las que se presentan a continuación.

"Claro que sí, las estrategias preinstruccionales si las utilizo, porque cómo hacen ellos algo si uno no les da las instrucciones para que trabaje" (docente 2).

"Si. Yo siempre les pregunto a mis alumnos qué quieren aprender ese día. Tú sabes, por eso de los proyectos pedagógicos de aula" (docente 4).

Estas afirmaciones evidencian que el docente concibe a las estrategias preinstruccionales como aquellas que se limitan a informar al alumno como se hacen las actividades y no a indagar posibles informaciones y conocimientos del alumno relacionados al tema en estudio, ni para despertar en los alumnos el interés ante el nuevo tema que se le presenta y prepararlo adecuadamente para enfrentarse a la nueva experiencia. Ya que como lo señala Gagné (1987), todo nuevo aprendizaje pasa a ser una experiencia nueva.

En relación a las estrategias coinstruccionales, las cuales permiten que el alumno empiece a descubrir relaciones y similitudes entre las partes aisladas presentadas o estudiadas con antelación, llegando a configurar esquemas y mapas cognitivos sobre los contenidos desarrollados como lo señala Sánchez (1999), alcanzando así aprendizajes en forma progresiva y paulatina, lo cual permite que el conocimiento aprendido se vuelva aplicable a otros contextos, se extrajeron de las entrevistas realizadas los siguientes fragmentos 
La praxis educativa: una aproximación a la realidad en el aula

Castro, Elizabeth; Peley, Rosario y Morillo, Roselia

"La coinstruccional es sobre lo que vamos a trabajar, y sí claro, siempre la debo utilizar" (docente 7).

"Hay veces que uno no las aplica pero ellos mismos las buscan" (docente 4).

"Yo utilizo estrategias coinstruccionales semanalmente, o quizás todos los días un poquito, pero no puede ser todos los días, porque ellos como que no le tomarían seriedad o responsabilidad a las actividades" (docente 8).

"Con estrategias coinstruccionales sería un poco difícil enseñarles lenguaje, en el aspecto de que ellos son tantos niños que para evaluarlos individualmente sería difícil saber quien aprendió, y quien no, por eso es que yo creo que sería difícil" (docente 11).

Con estas afirmaciones se pudo evidenciar que los docentes desconocen las estrategias coinstruccionales, al opinar que ellos pueden desarrollar una clase sin utilizarlas; los fragmentos de las entrevistas también permitieron develar como los docentes subestiman la importancia que estas estrategias ejercen en el logro de aprendizajes permanentes en los alumnos, y la ayuda que proporcionan al docente para que la clase no sea un contenido visto sino que se convierta en una verdadera praxis educativa, permitiendo al educando ver la aplicabilidad o significatividad del conocimiento obtenido, y no ver la utilización de las estrategias coinstruccionales como una invitación a los alumnos para el desorden o al irrespeto por lo tratado durante el desarrollo de la clase.

Las opiniones de los docentes hacia el uso en sus clases de estrategias postinstruccionales, a través de las cuales el docente logra integrar los diferentes contenidos desarrollados en momentos anteriores de la clase, logrando así que los alumnos lleguen a desenvolverse con mayor autonomía y confianza, al obtener la integración entre el nuevo contenido y lo familiar, también fueron consideradas en la investigación, obteniéndose las siguientes.

"Sí, yo utilizo las estrategias postinstruccionales, porque esas son las actividades que llevan para con los padres, yo les informo como la van a manejar con sus papas a duras penas" (docente 2).

"Siempre que les doy una clase les coloco actividades, tareas, las cuales considero estrategia postinstruccionales" (docente 7).

Al analizar las respuestas de los docentes hacia el uso de las estrategias postinstruccionales, se observa que los docentes limitan el final de sus clases a indicar a los alumnos las actividades o tareas a realizar luego, desplazando con este tipo de práctica docente lo importante de recapitular lo tratado a lo largo de la clase, contribuyendo a afianzar en los alumnos los procesos y aspectos desarrollados durante la misma.

La categoría estrategias instruccionales utilizadas por los docentes, permitió conocer el tiempo que los docentes dedican en la ejecución de estrategias preinstruccionales, coinstruccionales y postinstruccionales, lográndose asi obtener no sólo la clasificación de las estrategias instruccionales utilizadas en la actualidad por los docentes que constituyeron la muestra, sino también el tiempo de ejecución de las mismas, aspecto de gran importancia en el desarrollo de toda clase.

Se considera que para que una clase pueda ser desarrollada adecuadamente, se deben utilizar las estrategias 
pertinentes a cada momento con un tiempo de ejecución adecuado, es decir, que no se puede afirmar que la clase ha sido exitosa por el hecho de desarrollar en esta las estrategias correspondientes a cada uno de sus momentos, es necesario además, establecer y respetar el tiempo de ejecución de todas y cada una de las estrategias utilizadas a lo largo de la clase, contribuyendo a obtener con este tipo de ejecución y dedicación de las estrategias preinstruccionales, coinstruccionales y postinstruccionales, el desarrollo pleno de habilidades y destrezas en los alumnos, considerando que para que las habilidades y destrezas puedan desarrollarse se debe trabajar en el alumno los procesos, los cuales pueden emerger con la utilización de estrategias instruccionales en los momentos de la clase y con la dedicación que estos requieren durante su desarrollo.

Considerando como señalan Díaz y Hernández (2001: 27), que "el aprendizaje se facilita cuando los contenidos se le presentan al alumno organizados de manera conveniente y siguen una secuencia lógica-psicológica apropiada", es decir, que es conveniente delimitar a través de las estrategias instruccionales intencionalidades y contenidos de aprendizaje en una progresión continua que respete niveles de inclusividad, abstracción y generalidad, contribuyendo así a desarrollar en el alumno sus potencialidades, sus habilidades y destrezas.

Los resultados obtenidos a través del guión de observación permiten afirmar que el $92.85 \%$ de los docentes utilizan las estrategias instruccionales en un momento de la clase, es decir, que los docentes no le dan la debida impor- tancia a la ejecución de estrategias instruccionales durante todos los momentos de la clase. Aspecto considerado por Díaz y Pacheco (1998), como fundamental para que el docente pueda obtener resultados positivos durante su práctica pedagógica. Los autores mencionados señalan, que los docentes obtendrán respuestas satisfactorias de sus alumnos si se preocupan en aplicar las estrategias adecuadas en todos y cada uno de los momentos de la clase, aspecto este que contribuye a lograr en el alumno un aprendizaje significativo.

La misma categoría, estrategias instruccionales utilizadas por los docentes de la primera etapa de educación básica, permitió develar que la pertinencia de las estrategias instruccionales utilizadas en relación a los contenidos fue la siguiente.

Se observó que el $50 \%$ de los docentes logró adecuar las estrategias instruccionales al contenido y asignatura en estudio, evidenciándose que el otro $50 \%$ de los docentes, no estableció correspondencia entre el contenido de la asignatura y la naturaleza de la estrategia instruccional desarrollada durante la clase, obviando con esa manera de ejecutar sus clases el vínculo entre las estrategias instruccionales y el contenido a desarrollar que según Díaz y Hernández (2001), no debe ser roto por muy complicado que este sea (contenido a desarrollar), es decir, que el docente debe valerse de diversos medios y estrategias instruccionales para lograr que los contenidos a tratar con sus alumnos puedan ser aprendidos de la mejor manera posible. Si no se adecuan las estrategias instruccionales al contenido en cuestión, se corre el riesgo de que este no 
La praxis educativa: una aproximación a la realidad en el aula Castro, Elizabeth; Peley, Rosario y Morillo, Roselia

sea captado de la mejor manera por los alumnos, perdiéndose así toda oportunidad de desarrollar una clase satisfactoria tanto para el docente como para el alumno, dejándose de propiciar en la práctica pedagógica un aprendizaje para la vida, un aprendizaje significativo.

En lo que respecta al dominio de las estrategias instruccionales utilizadas por los docentes durante el desarrollo de sus clases, se obtuvieron los siguientes resultados. El $7.14 \%$, es decir, sólo uno de los docentes, ejecuto adecuadamente las estrategias instruccionales en cada uno de los momentos de la clase, demostrando dicho porcentaje que la mayoría de los docentes observados, desconocen las estrategias instruccionales; el otro $92.85 \%$ no manejó adecuadamente las estrategias instruccionales desarrolladas a lo largo de sus clases.

Se puede observar a través del guión de observación, que el porcentaje de docentes que combinó estrategias instruccionales durante el desarrollo de la clase para lograr así un mejor rendimiento por parte de sus alumnos es de $28.57 \%$, porcentaje que evidencia la poca importancia que brinda el docente al hecho de facilitar a los alumnos los contenidos, tópicos y temas a través de la implementación de diversas estrategias instruccionales, denegándose así la oportunidad de acercarse a todos sus alumnos, obviando el supuesto de que todos los alumnos no aprenden a la ves, ni con la utilización de los mismos sentidos, como lo manifiesta Sánchez (1999:112) al afirmar ..."el docente debe buscar la excelencia cognoscitiva, a través de la utilización de la mayor cantidad de estrategias que permitan desarrollar habilidades $y$ destrezas", es decir, se trata de emplear diversas estrategias instruccionales, que les permita a los alumnos encontrar la vía más expedita para comprender y asimilar contenidos, relacionarse con el entorno, resolver problemas, tanto cotidianos como trascendentales.

A través del guión de observación se evidenció que el $50 \%$ de los docentes mostró una conducta proactiva y dinámica durante la ejecución o desarrollo de sus clases, es decir, que el otro $50 \%$ pareciera no conocer, o no darle importancia a la creciente interacción existente entre la escuela y la sociedad, influyendo cada vez más en el rol del docente. Donde la tarea del docente debe dirigirse fundamentalmente hacia el alumno y su desarrollo personal y social, lo cual trae como resultado que su labor se diversifique cada día más. Reafirmando que para que exista una adecuada interacción, el docente debe ser proactivo y dinámico, permitiéndose actuar como mediador en el proceso de aprendizaje de los alumnos de manera pertinente.

El hecho de ser un docente proactivo y dinámico contribuye a propiciar el proceso motivacional en los alumnos, aportar criterios y diagnosticar situaciones de aprendizajes de cada alumno en particular y del conjunto de la clase. Al igual que le permitirá innovar en la utilización de recursos y medios, clarificar y aportar valores, ayudar a que los alumnos desarrollen los suyos propios, y algo muy importante contribuirá a promover y facilitar las relaciones humanas durante las clases y el entorno del alumno, logrando que el alumno genere con ese tipo de práctica docente el desarrollo de sus habilidades y destrezas. 
Sin embargo cuando se encuestó a los docentes, estos coincidieron en un $68.28 \%$ en que la motivación siempre estaba presente en su trabajo, el otro $28.57 \%$ manifestó que frecuentemente estaban motivados y sólo un $7.14 \%$ que su motivación era en ocasiones, es decir, a veces, al analizar las respuestas de los docentes en el cuestionario se evidencia una contradicción, ya que los docentes manifestaron casi en su totalidad sentirse motivados y en las observaciones de sus clases se obtuvo que sólo el $50 \%$ del total de los docentes mostró una conducta proactiva y dinámica.

Relacionado a la conducta proactiva y dinámica de los docentes y a su motivación, en la entrevista a profundidad se obtuvieron respuestas como la siguiente.

"Mantenerse motivada cuando uno trabaja en aula no se logra muy fácil, las deficiencias de los niños, su pobreza, sus problemas, y uno sin poder hacer nada, porque tu sabes el sueldo de uno tampoco da para mucho, a pesar de que nos han mejorado un poco en estos últimos años" (docente 1).

Al analizar este tipo de afirmaciones se refuerza la contradicción ya antes descrita, ya que un $96.85 \%$ de los docentes manifestaron estar siempre y frecuentemente motivados y su actuación y respuestas en las entrevistas muestran otro tipo de información como se presenta a continuación.

"En ocasiones me siento motivada, con ganas de trabajar, sobre todo cuando venimos de vacaciones, pero cuando uno empieza a trabajar lo que encuentra es problemas y problemas, ya los alumnos no son como antes, yo muchas veces no se que hacer con ellos y eso desmotiva a cualquiera, es que mira educar no es fácil" (docente 13).

Otro aspecto que permitió develar la opinión de los docentes estuvo constituido por el conocimiento, específicamente el conocimiento que deben tener los docentes en lo que respecta al uso adecuado de las estrategias instruccionales, obteniéndose los siguientes resultados.

Del total de docentes encuestados el $85.71 \%$ manifestaron sentirse siempre capacitados para utilizar materiales didácticos en el aula, y un $78.57 \%$ para planificar y desarrollar actividades innovadoras para la ejecución de sus clases. Como se puede observar los porcentajes son semejantes, al igual que en lo que respecta a estrategias instruccionales que permitan mantener la disciplina y la organización del tiempo y el espacio en la clase. Los docentes manifestaron estar siempre, y casi siempre, preparados en estos dos aspectos.

El conocimiento también pudo ser abordado mediante la aplicación del guión de observación, a través del cual se constató que los docentes aplican cada uno de los pasos de las estrategias instruccionales seleccionadas por ellos en un $7.14 \%$, es decir, que el otro $92.85 \%$ no desarrollaron adecuadamente las estrategias instruccionales durante sus clases.

Es importante destacar que sólo el $7.14 \%$ de los docentes, es decir, solamente uno de ellos demostró conocer y dominar las estrategias instruccionales. Los resultados del guión de observación permitieron afirmar que los docentes no se preocuparon en desarrollarlas o ejecutarlas pertinentemente, es decir, que los docentes en su mayoría no mostraron dominio o conocimiento de las estrategias 
La praxis educativa: una aproximación a la realidad en el aula Castro, Elizabeth; Peley, Rosario y Morillo, Roselia

instruccionales utilizadas, al igual que desinterés en aplicarlas.

Los docentes manifestaron en el cuestionario que se sentían capacitados para utilizar materiales didácticos en un $85.71 \%$, y que planificaban sus actividades de manera innovadora en un $78.57 \%$, lo cual les permite desarrollar las clases de manera pertinente, ayudándoles a obtener por parte de los alumnos los resultados esperados.

Lo cual evidencia una contradicción entre lo que los docentes expresaron en el cuestionario y lo observado por el investigador durante las clases, ya que los docentes manifestaron sentirse capacitados para utilizar materiales didácticos, que planificaban sus clases de manera innovadora, y que en la organización del tiempo y espacio de sus clases se sentían capacitados, aspectos estos que no son posibles desarrollar si no se ejecutan las estrategias instruccionales pertinentes en lo que respecta a los diferentes pasos, fases y momentos de la clase.

\section{- Categoría: Planificación Instruc- cional}

Al consultar a los docentes en el mismo cuestionario si utilizaban estrategias actualizadas en la planificación de sus clases, los resultados fueron un $71.42 \%$ siempre las utilizan y un $28.57 \%$ casi siempre las utilizan, con estos porcentajes no sólo se corrobora que el docente utiliza en sus planificaciones estrategias instruccionales actualizadas, sino también que utilizan las técnicas de los Proyecto Pedagógico de Aula, ya que estas forman parte del conjunto de estrategias actualizadas, sin embargo para reforzar estos resultados se compararon con las respuestas que los docentes dieron en la entrevista a profundidad. Al respecto se transcriben fragmentos de la entrevista.

"Bueno trabajamos con proyectos, y las estrategias que más utilizo es la comunicación directa con el niño, observaciones que voy utilizando diariamente y las pruebas, evaluaciones que se les hacen semanalmente, dependiendo el material que utilizo durante la semana, lo evalúo al final de la semana" (docente 5).

Con estas afirmaciones los porcentajes antes descritos relacionados a la utilización de las técnicas sugeridas por los Proyecto Pedagógico de Aula y si utilizan estrategias actualizadas en la planificación de sus clases dejan de ser significativos, ya que en la entrevista a profundidad los docentes expresaron que tienen pocos conocimientos relacionados a los Proyecto Pedagógico de Aula, lo cual incide en su poca confianza ante estos proyectos, y la certeza de algunos de ellos de creer que con los Proyecto Pedagógico de Aula sus alumnos no aprenden, es decir, que la contradicción entre los resultados del cuestionario, el guión de observación y la entrevista a profundidad es evidente.

En relación a la planificación de las clases, el cuestionario arrojo que el $78.57 \%$ de los maestros encuestados siempre tienen tiempo para planificar, un $14.28 \%$ frecuentemente y un $7.14 \%$, es decir, un docente a veces tiene tiempo de planificar, información que fue corroborada al realizar las observaciones, en la cual se constato que los docentes en un $85.71 \%$ elaboraron el proyecto pedagógico de aula, sin embargo, en las observa- 
ciones realizadas a los mismos docentes se constato que un $21.42 \%$ de los docentes se guió por los proyectos antes elaborados, con esto se evidencia que no todas las planificaciones realizadas por los maestros son desarrolladas luego, sin embargo, al consultar en el cuestionario si aplicaban en sus clases la planificación realizada, el $85.57 \%$ de los docentes contestaron que siempre lo hacían y un $14.28 \%$ que frecuentemente, estos dos datos refuerzan que el docente en un $100 \%$ utiliza siempre y casi siempre la planificación que realiza, sin embargo, estos datos se contrastaron con las siguientes afirmaciones de los docentes durante la entrevista a profundidad.

"Yo he realizado Proyecto Pedagógico de Aula que después que termino no me gustan, se los muestro a la directora y me dice que lo mejore, entonces para que aplicarlos si no están bien, prefiero aprovechar el tiempo en reforzar lectoescritura y matemáticas que están tan mal" (docente 8).

El desarrollo de las actividades planificadas en el tiempo previsto por los docentes reveló que el $85.71 \%$ de los docentes afirmaron ejecutar siempre las actividades planificadas previamente por ellos, y el otro $14.28 \%$ restante afirmaron que frecuentemente, estos datos permiten concluir que todos los docentes encuestados desarrollan de manera continua las actividades reflejadas en sus planificaciones, sin embargo es propicio mencionar que en las observaciones realizadas por el investigador en las diferentes clases administradas por los docentes, los resultados fueron los siguientes. Del total de docentes observados, un $71.42 \%$ no se guiaron por un plan preela- borado, es decir, que sólo a un $21.42 \%$ de los docentes se les observó una planificación, y lo más importante que se guiaran por ella.

A través del guión de observación, se evidenció que los docentes si realizan sus planificaciones, sus Proyectos Pedagógicos de Aula, pero no los ejecutan luego, es decir, que diseñan proyectos pedagógicos de aula con sus respectivos diagnósticos, contenidos, competencias, ejes transversales entre otros, pero luego los desplazan o dejan de desarrollarlos por considerarlos pocos productivos, es decir, que en ocasiones los docentes se limitan a planificar sus Proyectos Pedagógicos de Aula por cumplir con el mero requisito de planificar, lo cual se confirma con los siguientes fragmentos de la entrevista a profundidad.

"No puedo pasar el año a fuerza de proyectos porque entonces nunca aprenden a leer, tu sabes a los alumnos hay que enfatizarles mucho la lectura, hasta el cansancio diría yo y eso no se puede con los proyectos, bueno yo no he podido, y creo que aquí nadie" (docente13).

"Enseñar a leer y matemáticas a fuerza de proyectos no es fácil, yo prefiero el otro método, el de antes, me siento mejor trabajando como antes y veo que los muchachos aprenden más, mira que los alumnos no aprenden a leer si no es por sonidos y deletreando, y eso que el ministerio dice que así no debe ser pero ellos mismos buscan esa manera, ¿como te digo? A ellos les gusta es así, y eso con los proyectos no se puede porque no da tiempo" (docente 9).

"Yo empiezo el año con proyectos, pero a la mitad tengo que trabajar con reforzamientos, porque hay muchos retrasa- 
La praxis educativa: una aproximación a la realidad en el aula Castro, Elizabeth; Peley, Rosario y Morillo, Roselia

dos y tengo que ayudarlos a recuperar porque si no se quedan" (docente 3 ).

Luego de conocer estas opiniones, es oportuno mencionar, que si los docentes desarrollaran sus actividades en el tiempo previsto, no tendrían planificado Proyectos Pedagógicos de Aula a desarrollar en un tiempo estipulado de tres meses y que luego dicen cubrir en un mes por considerar que los alumnos no aprenden con los Proyectos Pedagógicos de Aula como lo han expresado durante la entrevista a profundidad.

La entrevista permitió conocer las siguientes afirmaciones relacionadas a la planificación.

"Uno necesita más tiempo en el sentido de planificar uno, dedicarse un poquito más, no aquí dentro del aula, sino afuera. Uno tiene que tener un poco más de tiempo, pero uno sale de 3:30 a 4:00 PM para dedicarse a la familia. Es muy difícil" (docente 2).

"A nosotros nos han dado un poco de talleres acá, pero con muy poco tiempo, y esto de los proyectos, bueno será porque nosotros, en sí, yo no entiendo en verdad. Porque nosotros preparamos el proyecto, las actividades, las estrategias, los ejes transversales, y supuestamente la directora nos da una información. Nos revisa el proyecto y no ve fallas" (docente 5).

"Muy poco nos han dado capacitación para los Proyectos Pedagógicos de Aula, muy poco. Entonces siempre quedan a media, pero en verdad si nosotros queremos realizar un proyecto bueno, tenemos que tener antes un buen taller que nos enseñe" (docente 11).

"Bueno, estos Proyectos Pedagógicos de Aula traen una cosa negativa. ¿Tú te diste cuenta del examen que yo les hice? Ellos muy poco saben. La evaluación es muy poca. Porque tú sabes, ahora con la planificación por proyectos las actividades y los recursos que más utilizamos son las participaciones, el interrogatorio, las observaciones que hacemos cuando estamos hablando. Más que todo, las participaciones, cuando el niño participa. Pero en la elaboración de una evaluación, eso si que estoy yo. Yo no se si es falla mía, o de los niños o de los mismos proyectos. Porque fíjate que yo los tenía desde segundo grado, y yo les había hecho una evaluación así, y fíjate que ellos oral lo recalcaron todo, pero yo no se por qué por escrito la cosa está tan mal, esta planificación me cuesta, me cuesta mucho en verdad" (docente 15).

"No se. Yo no me adapto mucho a eso de los proyectos. Por cierto, nosotros hicimos unos proyectos, y la supervisora los revisó y sacamos unas cuantas observaciones porque no estaban bien, $y$ eso que nos lo ayudaron a hacer profesoras de La Universidad del Zulia" (docente 9).

"Yo soy planificada, claro a veces no me da tiempo planificar y me pongo a ver en el salón qué les doy. Pero si, siempre hay que planificar" (docente 6).

"Es que sólo el curso del ministerio lo hizo la primera etapa, la segunda no. Lo hicimos en escuelas diferentes, y a todos nos dieron cosas diferentes" (docente 5).

Estas opiniones discrepan con las obtenidas en el cuestionario, las cuales dejan ver la poca formación que poseen los docentes que constituyeron la muestra en relación a la planificación a través 
de los Proyecto Pedagógico de Aula; y algo muy importante, el desinterés de ellos hacia la ejecución de este tipo de estrategias en la planificación y desarrollo de sus clases.

\section{- Categoría: Responsabilidad del docente}

La observación efectuada permitió conocer si la actitud de los docentes era responsable, obteniendo como resultados los siguientes, el $92.85 \%$ de los docentes mostraron responsabilidad, la cual fue considerada por el investigador, a través de la asistencia de los docentes a la institución, la hora de llegar e iniciar sus clases, la entrega a tiempo de sus planificaciones entre otras, si se observa la responsabilidad de los maestros hacia los aspectos mencionados es bastante alta, positiva, aun y cuando en los otros ítems se ha constatado que el docente sólo se limita a realizar y entregar las planificaciones, pero no a desarrollarlas y a darle continuidad de manera creativa, aspecto que también fue abordado en la investigación a través del cuestionario.

\section{- Categoría: Comunicación}

La comunicación también fue abordada con el fin de conocer si los docentes utilizaban en sus clases estrategias instruccionales que le permitieran comunicarse con los alumnos, para lo cual los docentes respondieron en un $64.28 \%$ que siempre hacían uso de ese tipo de estrategias instruccionales, un $28.57 \%$ frecuentemente y el otro $7.14 \%$ a veces, estos resultados permiten afirmar que un $92.85 \%$ afirmaron hacer uso de dichas estrategias.

Con respecto a la comunicación también se consulto a los docentes si lograban establecer una comunicación abierta con los alumnos, afirmando los docentes en un $92.85 \%$ que siempre logran establecer una comunicación abierta y un $7.14 \%$ frecuentemente, es decir, que los docentes en un $100 \%$ sienten que logran establecer una pertinente comunicación con sus alumnos.

El tema de la comunicación también se abordo a través del guión de observación obteniéndose los siguientes resultados, el $64.28 \%$ de los docentes observados logran crear un clima adecuado para que se consoliden las comunicaciones abiertas, el $21.42 \%$ no, el $78.57 \%$ de los docentes escucha a sus alumnos, el $21.42 \%$ no, con esto se evidencia que los resultados obtenidos en el cuestionario no son confiables, ya que los docentes afirmaron en un $100 \%$ comunicarse abiertamente con sus alumnos y en las observaciones se constato que sólo un $64.28 \%$ logra crear un clima propicio para las comunicaciones y un $78.50 \%$ de los docentes escucha a sus alumnos, no se puede hablar de adecuadas comunicaciones si no se les da a los alumnos la oportunidad de comunicarse.

\section{- Categoría: Retroalimentación}

Otro aspecto considerado en las observaciones fue el tipo de retroalimentación que ofrecen los docentes a sus alumnos, para lo cual se observó que un $14.28 \%$ de los docentes retroalimentó a sus alumnos, preocupándose por elogiarlos o corregirlos según lo ameritara la situación, tratando en los casos de consultas, preguntas o dudas de constatar si el alumno había esclarecido sus inquietudes o no, el otro $85.71 \%$ no se preocupó por retroalimentar a los alumnos, aun y cuando estuviera de acuerdo con sus opi- 
La praxis educativa: una aproximación a la realidad en el aula Castro, Elizabeth; Peley, Rosario y Morillo, Roselia

niones y comentarios, es decir, estos docentes no utilizaban los elogios ni preguntas indagatorias durante el desarrollo de la clase.

\section{- Categoría: Participación}

La participación de los alumnos también fue utilizada para conocer la importancia que los docentes le brindan a esta dentro del salón de clases. Obteniéndose los siguientes resultados.

El $71.42 \%$ de los docentes manifestaron crear un ambiente de aprendizaje propicio para el desarrollo de sus clases, y el $21.42 \%$ afirmaron lograrlo frecuentemente, al analizar estos resultados se puede afirmar que los docentes en un $100 \%$ desarrollan sus clases en ambientes adecuados para el aprendizaje, característica indispensable para que se pueda desarrollar un verdadero aprendizaje, como lo señala González (2000), un clima adecuado dentro del aula es el primer paso para iniciar el aprendizaje académico. Cuando una persona se siente cómoda, en confianza, en otras palabras motivada y tiene la sensación de que lo que está haciendo tiene sentido y significado, obtiene mejores resultados, es decir, que un gran porcentaje de los docentes encuestados reconocieron la importancia de gestar un ambiente propicio para el desarrollo de la clase, que invite al alumno a participar activamente en ellas.

Este aspecto también fue considerado en el guión de observación, obteniéndose los siguientes resultados, el $57.14 \%$ de los docentes observados lograron crear un clima adecuado que impulsara la participación de los alumnos a través del uso de dinámicas que permitieran hacer la clase interesante para los alumnos. Si se compara lo observado por el investigador en el desarrollo de las clases, con lo dicho por los docentes en el cuestionario se evidencia una contradicción, puesto que los docentes manifestaron crear ambientes adecuados en un $100 \%$ y sólo se les observó realizar actividades que conducen a hacer la clase agradable e interesante al $57.14 \%$ de ellos, es decir, que un $42.85 \%$ de los docentes no se preocuparon por iniciar, mantener y dirigir el entusiasmo y la perseverancia de los alumnos hacia el logro de los objetivos.

\section{Reflexiones Finales}

Las reflexiones finales fueron organizadas tomando como criterio las categorías diseñadas para el estudio.

La investigación permitió conocer, que los docentes no se preocupan por mejorar la calidad de la educación y permitir que los alumnos aprendan significativamente, en virtud de la baja motivación que presentan hacia su labor. Sólo alcanzan, con las estrategias instruccionales utilizadas, cubrir algunos aspectos propuestos por el CBN, sin preocuparse en demostrar si los aprendizajes desarrollados en el aula son verdaderamente trascendentes para el alumno.

El docente basa su práctica pedagógica en la utilización de estrategias instruccionales que despiertan muy poco el deseo de aprender y crear de manera innovadora y original, asimismo, no promueven durante el desarrollo de sus clases la participación activa y consciente de sus alumnos. No logran en sus praxis una comunicación abierta con sus alumnos, 
generando en muchos casos un clima tenso en el aula.

Por otra parte, se evidenció que la vinculación entre la información existente en el alumno y la nueva no se establece, coartando al alumno de establecer vínculos entre sus conocimientos y los nuevos, al igual, que entre su entorno y sus nuevos conocimientos, supuestos que contribuirían a acrecentar en el alumno no sólo su identidad, sino también su importancia dentro de la escuela y comunidad.

Con una práctica docente de espaldas a las condiciones y realidades del entorno es difícil lograr que los alumnos desarrollen sus habilidades y destrezas cognoscitivas, es decir, que los docentes a lo largo de la investigación develaron que utilizan muy poco estrategias instruccionales que fomenten habilidades y destrezas cognoscitivas, al obviar en la práctica la educación centrada en procesos, tan importantes por considerárseles componentes activos de la mente, y por lo tanto son elementos básicos para construir, organizar y usar los conocimientos.

\section{Referencias Bibliográficas}

Ausubel, David (1983). Psicología evolutiva. Un punto de vista cognitivo (2a. ed.). México: Editorial Trillas.
Castillo, Emilia (1996). La formación de formadores dinámicos. México: Editorial Trillas, segunda edición.

Delval, Juan (1997). Tesis sobre el constructivismo. En M. Rodrigo y J. Arnay (Comps.). La construcción del conocimiento escolar. Madrid. Ediciones Paidós Ibérica, S.A.

Díaz, Frida; Hernández, Gerardo (2001). Estrategias docentes para un aprendizaje significativo: Una interpretación constructivista. México: Mc Graw Hill.

Díaz, Frida; Pacheco, Lule (1989). Efectos de las estrategias preinstruccionales en alumnos de secundaria de diferentes niveles socioeconómicos. México: UNAM.

Gagné, Roger (1987). Las condiciones del aprendizaje. México: Interamericana.

González, Raiza (2000). Factores que inciden en la aplicación de estrategias docentes para el aprendizaje significativo del alumno de educación básica. Maracaibo: Tesis de doctorado no publicada, Universidad del Zulia, Maracaibo.

Nérice, Imideo (1980). Hacia una didáctica general dinámica. Buenos Aires: Editorial Kapelusz.

Rivero, José (1995). La educación y la crisis latinoamericana. Lima, Perú: Tarea.

Sánchez, Margarita (1999). Transferencia de los procesos de pensamiento a la enseñanza y al aprendizaje. México: Editorial Trillas. 